\title{
Chlorine Adsorption on Graphene: Chlorographene
}

\author{
H. Şahin ${ }^{\dagger, \ddagger, \perp}$ and S. Ciraci* ${ }^{\dagger} \dagger, \S$ \\ ${ }^{\dagger}$ UNAM-National Nanotechnology Research Center, ${ }^{\ddagger}$ Institute of Materials Science and Nanotechnology, and ${ }^{\S}$ Department of \\ Physics, Bilkent University, 06800 Ankara, Turkey
}

\begin{abstract}
We perform first-principles structure optimization, phonon frequency, and finite temperature molecular dynamics calculations based on density functional theory to study the interaction of chlorine atoms with graphene predicting the existence of possible chlorinated graphene derivatives. The bonding of a single chlorine atom is ionic through the transfer of charge from graphene to chlorine adatom and induces negligible local distortion in the underlying planar graphene. Different from hydrogen and fluorine adatoms, the migration of a single chlorine adatom on the surface of perfect graphene takes place almost without barrier. However, the decoration of one surface of graphene with $\mathrm{Cl}$ adatoms leading to various conformations cannot be sustained due to strong $\mathrm{Cl}-\mathrm{Cl}$ interaction resulting in the desorption through the formation of $\mathrm{Cl}_{2}$ molecules. On

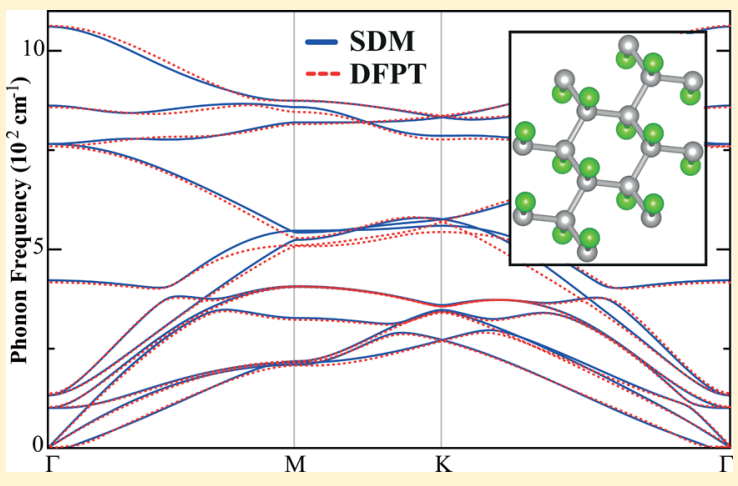
the contrary, the fully chlorinated graphene, chlorographene $\mathrm{CCl}$, where single chlorine atoms are bonded alternatingly to each carbon atom from different sides of graphene with $\mathrm{sp}^{3}$-type covalent bonds, is buckled. We found that this structure is stable and is a direct band gap semiconductor, whose band gap can be tuned by applied uniform strain. Calculated phonon dispersion relation and four Raman-active modes of chlorographene are discussed.
\end{abstract}

\section{INTRODUCTION}

The synthesis of graphene ${ }^{1,2}$ has led to intense research activity in the field of graphene-based nanoscale devices. Although graphene is one of the most mechanically strong materials having a wide range of extraordinary properties, ${ }^{1-5}$ practical device applications are limited by its metallic behavior and sensitivity to surface adsorbates.

Efforts to synthesize chemically modified graphene composites with tailored electronic, optical, and chemical properties have presented new directions in graphene research. In particular, band gap engineering of graphene through chemical modification, such as oxygenation ${ }^{6-11}$ and hydrogenation, ${ }^{12-17}$ is appealing for electronic applications, since the scalable fabrication of graphene-based devices without disturbing the strong honeycomb lattice has become possible. However, due to the complex atomic structure of grapheneoxides ${ }^{7}$ (GOs) and thermal instabilities of hydrogenated graphenes $(\mathrm{CHs})$ even at low temperatures, ${ }^{12-14}$ the search for the novel graphene-based materials is still continuing.

Over the past three years experimental ${ }^{18-21}$ and theoreti$\mathrm{cal}^{22,23}$ studies have demonstrated that chemical conversion of graphene to fluorographene $(\mathrm{CF})$ is possible. In addition to early studies on the atomic composition and band structure of fluorocarbon materials, ${ }^{24,25}$ it was reported that the monolayer CF has quite different vibrational spectra and Raman characteristics as compared to hydrogenated graphene analogues. ${ }^{22}$ We also investigated the electronic and elastic properties of possible fluorinated graphene materials and attempted to clarify the discrepancy between theoretical and experimental results. ${ }^{23}$ Easy synthesis, high-quality insulating behavior, and extraordinary mechanical strength of fluorographene $(\mathrm{CF})$ have inspired intense research on other halogendecorated graphene derivatives.

In addition to three known derivatives of graphene (GO, $\mathrm{CH}$, and $\mathrm{CF}$ ) the successful synthesis of chlorinated graphene was also achieved very recently. ${ }^{26}$ It is experimentally demonstrated that nondestructive and patternable conversion of graphene is possible by using various photochemical chlorination techniques. ${ }^{26-28}$ While the research on chlorine-graphene interaction is rapidly growing, ${ }^{26-36}$ comprehensive research on the stability of various chlorinated graphene structures and their resulting properties is sparse.

In this paper we present a detailed analysis of the interaction between chlorine atom and graphene leading to the chlorination of graphene. Although the possibility of covering graphene surfaces with chlorine atoms has been reported, analyses of structural stability and electronic and magnetic properties as a function of $\mathrm{Cl}$ coverage are lacking. Our main motivation is to reveal which conformations of chlorinated graphene are stable and how these conformations modify the properties of graphene. To this end we investigated the chlorination of graphene starting from single $\mathrm{Cl}$ adsorption to full coverage leading to chlorographene, namely $\mathrm{CCl}$. At low coverage with diminishing $\mathrm{Cl}-\mathrm{Cl}$ coupling, the binding of $\mathrm{Cl}$ to graphene is significant, but adsorbed $\mathrm{Cl}$ atoms migrate on the surface of graphene almost without an energy barrier. We found

Received: July 15, 2012

Revised: October 2, 2012

Published: October 22, 2012 
that the nonbonding chair conformation of chlorographene $\mathrm{e}^{30,31}$ $(\mathrm{CCl})$ consisting of a planar graphene sandwiched between two widely spaced planar $\mathrm{Cl}$ layers is unstable. On the other hand, the covalently bonded chair conformation of the chlorographene $(\mathrm{CCl})$ is found to be stable at $T=0 \mathrm{~K}$ and possibly at room temperature. This latter conformation consists of buckled graphene sandwiched between two planar $\mathrm{Cl}$ layers and is a nonmagnetic semiconductor with a $1.2 \mathrm{eV}$ direct band gap. Our results reconcile the discrepancy between the experimental study $^{26}$ obtaining semiconducting properties upon the chlorination of graphene and theoretical studies predicting metallic state. ${ }^{30,3}$

\section{COMPUTATIONAL METHODOLOGY}

To investigate mechanical, electronic magnetic properties of chlorinated graphene we carried out first-principles density functional theory (DFT) calculations within the local density approximation (LDA) ${ }^{38}$ using projector augmented wave (PAW) potentials. ${ }^{37}$ All results discussed in the text are obtained by using LDA. To compare with the LDA results of specific systems, we also performed calculations using Generalized Gradient Approximation ${ }^{39}$ (GGA) together with van der Waals $(\mathrm{vdW})$ correction $(\mathrm{GGA}+\mathrm{vdW}) .{ }^{40}$ In our earlier tests LDA yielded interlayer spacings of layered materials and other structural parameters in agreement with experimental data, as well as with those obtained by using GGA+vdW. In the $\mathrm{vdW}$ corrections of the later method, DFT description is restricted to shorter correlation length scales, but for the medium and large interatomic distances the damped $\mathrm{C}_{6} r^{-6}$ term is used. The systems, whose numerical values are also obtained by using GGA+vdW, are indicated. Numerical calculations are performed by using VASP. ${ }^{41}$ The kinetic energy cutoff, $E_{\text {cut }}=$ $\hbar^{2}|\mathbf{k}+\mathbf{G}|^{2} / 2 m$, for the plane-wave basis set is taken as $500 \mathrm{eV}$. A vacuum spacing of at least $15 \AA$ is placed between adjacent layers to hinder the layer-layer interactions. The convergence criterion of self-consistent calculations for ionic relaxations is $10^{-5} \mathrm{eV}$ between two consecutive steps. Structural optimizations were performed by using a conjugate gradient algorithm with a convergence criterion of $10^{-4} \mathrm{eV} / \AA$. Pressures on the lattice unit cell are decreased to values less than $1.0 \mathrm{kBar}$. The adsorption of a single $\mathrm{Cl}$ atom to the graphene surface is treated by using the supercell geometry, where the single $\mathrm{Cl}$ is adsorbed to each $(4 \times 4)$ supercell. In the self-consistent potential and total energy calculations using a $(4 \times 4)$ supercell of chlorographene, a set of $(13 \times 13 \times 1)$ k-point sampling is used for Brillouin zone (BZ) integration. The sampling of BZ is then scaled according to the sizes of the supercells used for other systems. Ground state electronic structures are calculated by applying a dipole correction to eliminate the artificial electrostatic field between periodic supercells. For the charge transfer analysis, the effective charge on atoms is obtained by the Bader method. ${ }^{42}$

The stabilities of structures having various $\mathrm{Cl}$ coverage are examined by the calculation of phonon frequencies for $\mathbf{q}$-wave vectors over $\mathrm{BZ}$ by using both the small displacement method $(\mathrm{SDM})^{43}$ and density functional perturbation theory (DFPT) ${ }^{44}$ We used a 196-atom supercell of chlorographene, a q-point sampling grid of $3 \times 3 \times 1$, and $0.01 \AA$ displacements in calculations using SDM. The DFPT part of the phonon calculations was performed by using a $6 \times 6 \times 1$ grid of $\mathrm{q}$ points for the chlorographene unitcell. A given structure is considered to be stable if vibration frequencies are positive for all q-points in BZ.
The energy band gap, which is usually underestimated by DFT, is corrected by frequency-dependent $\mathrm{GW}_{0}$ calculations. ${ }^{45}$ In $G W_{0}$ corrections screened Coulomb potential $W$, is kept fixed to the initial DFT value $W_{0}$ and Green's function $G$ is iterated four times. Finally, the band gap of $\mathrm{CCl}$ is calculated by using $(12 \times 12 \times 1)$ k-points in $\mathrm{BZ}, 20 \AA$ vacuum spacing, a default cutoff potential for $G W_{0}, 160$ bands, and 64 grid points.

The binding energy of a single adatom $\mathrm{X}$ to the graphene supercell (i.e., $\mathrm{X}=\mathrm{H}$ hydrogenation; $\mathrm{X}=\mathrm{F}$ fluorination; $\mathrm{X}=\mathrm{Cl}$ chlorination) is calculated according to the following expression, $E_{\mathrm{b}}=E_{\mathrm{T}}[\mathrm{Gr}]+E_{\mathrm{T}}[\mathrm{X}]-E_{\mathrm{T}}[\mathrm{Gr}+\mathrm{X}]$, in terms of the ground state total energies ${ }^{46}$ of bare graphene $E_{\mathrm{T}}[\mathrm{Gr}]$, free $\mathrm{X}$ atom $E_{\mathrm{T}}[\mathrm{X}]$, and $\mathrm{X}$ adsorbed to graphene $E_{\mathrm{T}}[\mathrm{Gr}+\mathrm{X}]$. Accordingly, $E_{\mathrm{b}}>0$ indicates a bound state. Similarly, the formation energy of a single adatom adsorbed to a graphene supercell relative to the $\mathrm{X}_{2}$ molecule is defined as $E_{\mathrm{f}}=E_{\mathrm{T}}\left[\mathrm{X}_{2}\right] / 2$ $+E_{\mathrm{T}}[\mathrm{Gr}]-E_{\mathrm{T}}[\mathrm{Gr}+X]$, where $E_{\mathrm{T}}\left[X_{2}\right]$ is the ground state total energy ${ }^{46}$ of the $X_{2}$ molecule. For an exothermic process $E_{f}>0$. The cohesive energy of graphene fully covered with $\mathrm{X}, \mathrm{CX}$ relative to free $C$, and $X$ atoms is defined as $E_{\text {coh }}=2 E_{T}[X]+$ $2 E_{\mathrm{T}}[\mathrm{C}]-E_{\mathrm{T}}[\mathrm{CX}]$, where $E_{\mathrm{T}}[\mathrm{CX}]$ is the total ground state energy (per unit cell) of CX. By definition $E_{\text {coh }}>0$ indicates binding relative to individual constituent atoms.

\section{ADSORPTION OF A SINGLE CHLORINE}

Understanding of the adsorption process of a single $\mathrm{Cl}$ adatom on graphene is essential for the investigation of its chlorinated derivatives. The $\mathrm{Cl}-\mathrm{Cl}$ coupling between adsorbed $\mathrm{Cl}$ atoms is crucial. Here, the $\mathrm{Cl}$ coverage is defined as the ratio of the number of $\mathrm{Cl}$ atoms $N_{\mathrm{Cl}}$ to the number of carbon atoms $N_{\mathrm{C}}$ in a supercell; namely $\Theta=N_{\mathrm{Cl}} / N_{\mathrm{C}}$. A single $\mathrm{Cl}$ atom adsorbed to graphene is represented by a system where a $\mathrm{Cl}$ atom is adsorbed to each $(4 \times 4)$ supercell. This actually corresponds to a uniform coverage of $\Theta=0.03125$. On the basis of the analysis discussed in the next section we found the size of the $(4 \times 4)$ supercell is sufficient to neglect the $\mathrm{Cl}-\mathrm{Cl}$ coupling. Even if the Pauling scale of electronegativity of specific atoms alone cannot provide criteria for the character and strength of the bonds between those atoms, it usually indicates useful correlations. For example, according to the Pauling scale of electronegativity ( $\mathrm{H}: 2.20, \mathrm{C}: 2.55, \mathrm{Cl}: 3.16$, and $\mathrm{F}: 3.98$ ) the binding of $\mathrm{Cl}$ atoms to graphene can be expected to be stronger than that of hydrogen adatoms $(0.98 \mathrm{eV})$ and weaker than that of the fluorine adatoms $(2.71 \mathrm{eV})$. Three different adsorption sites can be foreseen for the adsorption of $\mathrm{Cl}$ on graphene as described in Figure 1a. These are the hollow $(\mathrm{H})$ site above the center of he hexagon, the top (T) site on top of the $\mathrm{C}$ atoms, and the bridge (B) site above the middle of the $\mathrm{C}-\mathrm{C}$ bonds. Among these sites, the strongest binding of single $\mathrm{Cl}$ to graphene is attained at the T-site with a binding energy of $E_{\mathrm{b}}=$ $1.10 \mathrm{eV}\left(E_{\mathrm{b}}\right.$ calculated by using GGA+vdW $\left.1.16 \mathrm{eV}\right)$. Earlier studies dealing with graphene- $\mathrm{Cl}$ interaction calculated the binding energies of the $\mathrm{Cl}$ adatom ranging between $1.05 \mathrm{eV}$, using the $(4 \times 4)$ supercell, and $0.8 \mathrm{eV}^{29}$ Using a methodology similar to that used in the present work the binding energy of $\mathrm{Cl}$ adatom is calculated to be $1.13 \mathrm{eV} .{ }^{36}$ Applying correction based on hybrid functionals ${ }^{47}$ and hence performing GGA +HSE06 calculations we found the binding energy of $\mathrm{Cl}$ as 0.7 $\mathrm{eV}$.

The variation of the total energy of an adsorbed $\mathrm{Cl}$ atom at the symmetry points and along the symmetry $(\mathrm{T}-\mathrm{B}-\mathrm{H}-\mathrm{T})$ directions of a hexagon in the $(4 \times 4)$ supercell is shown in Figure 1a. At each point on the energy curve, $x$ - and $y$-positions 

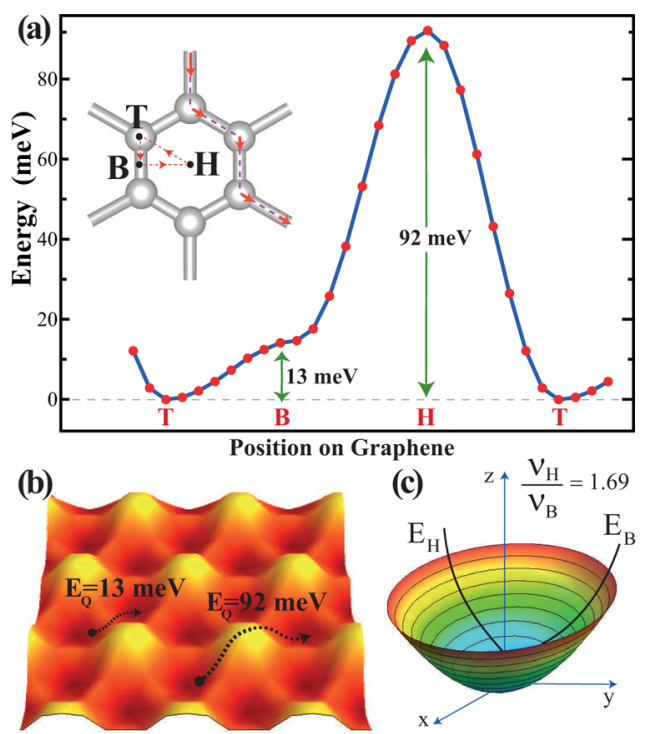

(d)

(e)

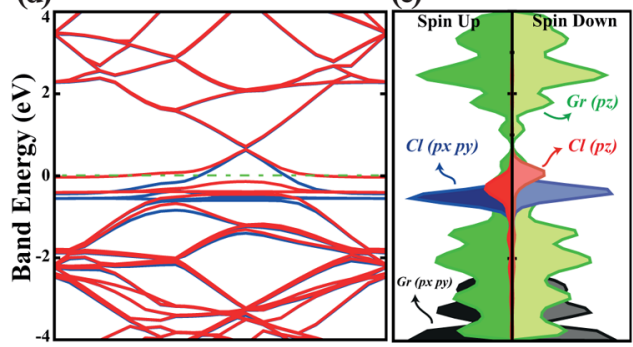

Figure 1. (a) Variation of energy for a single $\mathrm{Cl}$ adatom along the symmetry direction of a hexagon. Zero of energy is set to the energy of the $T$-site. The diffusion paths with the lowest energy barrier of $Q=13$ meV between two adjacent $\mathrm{T}$-sites are marked with thick red/dashed lines. (b) Energy landscape of a single $\mathrm{Cl}$ adatom adsorbed to graphene. Dark (light) colors represents the top (hollow) sites. (c) Potential energy contour plots (paraboloid) of $\mathrm{Cl}$ adatom around the $\mathrm{T}$-site. The jump frequencies of $\mathrm{Cl}$ atom $\nu$, for different directions are calculated from this paraboloid. (d) Band structure of a single $\mathrm{Cl}$ adsorbed to each $(4 \times 4)$ supercell of graphene and corresponding total (TDOS) and orbital decomposed (PDOS) densities of states. The zero of band energy is set to the Fermi level. Up-spin and downspin bands are shown in blue and red, respectively.

of the adsorbed $\mathrm{Cl}$ atom are fixed, its $z$-height, as well as positions of all $\mathrm{C}$ atoms in the $(4 \times 4)$ supercell are optimized by minimizing the total energy and atomic forces. Using this energy curve we reveal the energy barriers to be overcome by the adsorbate migrating or diffusing on the surface of graphene. The minimum energy barrier occurs at the B-site between two adjacent $\mathrm{T}$-sites. The relevant energy barriers are shown on the calculated energy landscaping presented in Figure $1 \mathrm{~b}$. This analysis suggests that a diffusing $\mathrm{Cl}$ atom can take a path of minimum energy barrier following the edges of the hexagon from T-site to T-site through the barrier of $Q=13 \mathrm{meV}$ at the B-site. This energy barrier is in fair agreement with that calculated by Wehling et al. ${ }^{29}$ We note that this barrier is very low and allows the $\mathrm{Cl}$ atom to migrate on the surface of graphene as if it rolls along the edges of the hexagon. This is a remarkable situation, where the migration or diffusion of $\mathrm{Cl}$ occurs with almost no barrier, but it remains bound to the surface. This property of the $\mathrm{Cl}$ adatom on graphene heralds a number of possible applications, such as superlow sliding friction, sensors, and devices for energy harvesting. ${ }^{34}$ The binding energy of an isolated $\mathrm{Cl}_{2}$ molecule is calculated to be
$3.58 \mathrm{eV},{ }^{48}$ leading to negative formation energy $E_{\mathrm{f}}<0$. Then the adsorption of chlorine on graphene is an endothermic process and hence it can be achieved by, for example, the assistance of light absorption. ${ }^{26}$

In Figure 1c the energy plot $E_{\mathrm{T}}(x, y)$ (or energy paraboloid) of the $\mathrm{Cl}$ adatom displacing from its equilibrium position at the $\mathrm{T}$-site is deduced approximately from the energy landscaping shown in Figure 1b. When displaced along $\mathrm{T}-\mathrm{H}$ and $\mathrm{T}-\mathrm{B}$ directions, the $\mathrm{Cl}$ adatom undergoes different energy variations. Using this energy plot and the model related to the simple harmonic oscillator we estimate the values of jump frequencies of the adatom toward $\mathrm{H}$ - and B-sites to be $\nu_{\mathrm{T} \rightarrow \mathrm{B}} \approx 0.97 \mathrm{THz}$ and $\nu_{\mathrm{T} \rightarrow \mathrm{H}} \approx 1.68 \mathrm{THz}$, respectively. The diffusion constant at a given temperature can be obtained from a simple expression, ${ }^{49}$ $D=\nu a^{2} \exp \left(-Q / k_{\mathrm{B}} T\right)$, in terms of energy barrier $Q$ lattice constant $a$, and the characteristic jump frequency $\nu$. Using these values the diffusion constant at $T=300 \mathrm{~K}$ is calculated for the barrier $Q=13 \mathrm{meV}$ to be $D=3.57 \mathrm{~cm}^{2} / \mathrm{s}$. This indicates that the $\mathrm{Cl}$ adatom migrates readily over the graphene layer.

The length of the $\mathrm{Cl}-\mathrm{C}$ bond is found to be $d_{\mathrm{Cl}-\mathrm{C}}=2.54 \AA$. This value is larger than the bond length calculated from the sum of covalent radii of $\mathrm{Cl}$ and $\mathrm{C}$ atoms, ${ }^{49}$ namely $r_{\mathrm{Cl}}+r_{\mathrm{C}}=$ $1.75 \AA$, as well as the bond lengths of $\mathrm{CCl}_{4}(1.77 \AA)$. This situation indicates that the character of this bond is rather different from the $\mathrm{C}-\mathrm{Cl}$ covalent bond in $\mathrm{CCl}_{4}$. When a single $\mathrm{Cl}$ is adsorbed to graphene, $0.44 e$ is transferred from graphene to the $\mathrm{Cl}$ adatom according to Bader analysis. ${ }^{42}$ The calculated charge transfer, as well as the analysis of charge density distribution, indicates an ionic character for the $\mathrm{Cl}-\mathrm{C}$ bond. ${ }^{36}$ The negative charge on $\mathrm{Cl}$ induces a dipole moment of $\mathbf{p}=0.67$ $e \cdot \AA$ when a single $\mathrm{Cl}$ atom is adsorbed to each $(4 \times 4)$ supercell of graphene. ${ }^{50}$ Consequently, the work function of bare graphene which is $4.49 \mathrm{eV}$ increases to $6.53 \mathrm{eV}$ upon $\mathrm{Cl}$ adsorption. This the energy required to remove an electron from the Fermi level to the vacuum energy at the side where $\mathrm{Cl}$ atoms are adsorbed. Incidentally, while the atomic configuration of graphene beneath the $\mathrm{Cl}$ adatom is rather flat, $\mathrm{H}$ and $\mathrm{F}$ adatoms on graphene impose buckling resulting in a covalent character. $^{14,23}$ As we will discuss in the next section, charge transfer from $\mathrm{C}$ to $\mathrm{Cl}$ and hence the character of the bonding in two sided adsorption is dramatically different.

While the single $\mathrm{Cl}$ adatom on the surface of graphene can be viewed as an impurity leading to localized (or resonance) states, our model representing the adsorption using a $(4 \times 4)$ supercell gives rise to an energy band structure, where dispersion of bands related to the adsorbate can be taken as the measure of adsorbate-adsorbate coupling. The band structure of a single $\mathrm{Cl}$ adsorbed to each $(4 \times 4)$ supercell of graphene presented in Figure 1d has filled chlorine states appearing as flat bands. In view of the negligible dispersions of these bands associated with $\mathrm{Cl}$ and the analysis of the bands corresponding to $(5 \times 5)$ and $(6 \times 6)$ supercells in the following section we concluded that a $\mathrm{Cl}$ adsorbed to each $(4 \times$ 4) supercell can mimic successfully the single, i.e. isolated $\mathrm{Cl}$ adsorbed to the graphene surface. Because of 7 valence electrons and hence a single unfilled $3 p$ orbital occurring below the Fermi level $E_{\mathrm{F}}$ of bare graphene, $\mathrm{Cl}$ adsorbed graphene is metalized and becomes magnetic with $\mu=0.56 \mu_{\mathrm{B}}$ due to broken spin degeneracy. The linearly crossing bands of bare graphene is raised above $E_{\mathrm{F}}$. The orbital decomposed density of states in Figure 1e shows that the flat bands below $E_{\mathrm{F}}$ are derived mainly from $\mathrm{Cl}-3 \mathrm{p}_{x}$ and $\mathrm{Cl}-3 \mathrm{p}_{y}$ orbitals. 


\section{COVERAGE OF GRAPHENE BY CHLORINE ADATOMS}

The interaction between two adsorbed $\mathrm{Cl}$ atoms as a function of their separation is important in understanding the coveragedependent properties and stability of $\mathrm{Cl}$-covered graphene. To this end we consider two $\mathrm{Cl}$ atoms adsorbed to the $(6 \times 6)$ supercell of graphene and calculate the total energies as a function of the separation between them. Chlorine atoms are placed at specific adsorption sites by fixing their $x$ - and $y$ coordinates, but their $z$-coordinates, as well as the positions of other $\mathrm{C}$ atoms in the supercell, are relaxed. In Figure 2 we show

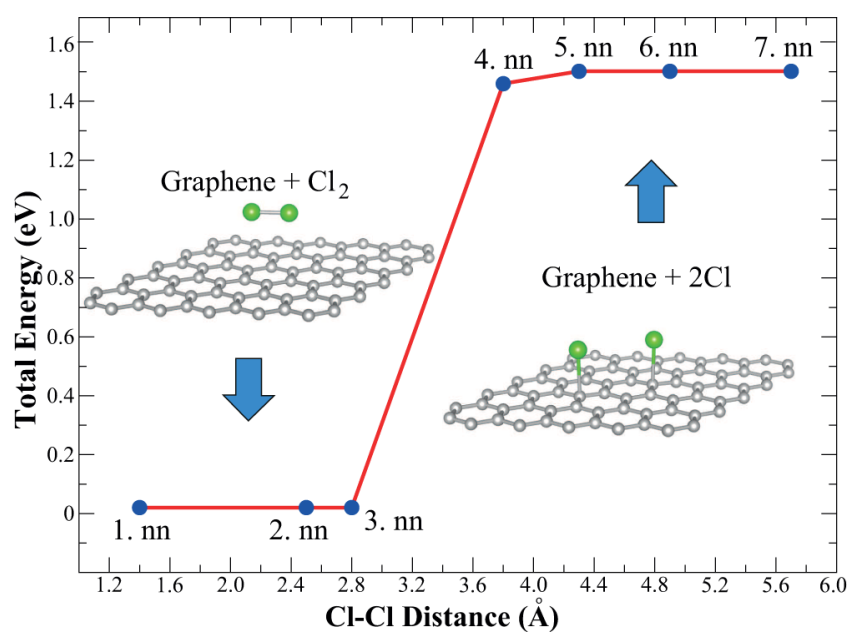

Figure 2. The interaction energy between two $\mathrm{Cl}$ atoms adsorbed to the same side of a $(6 \times 6)$ supercell of graphene. The zero of energy is set to the energy of $\mathrm{Cl}_{2}$ plus graphene. $\mathrm{nn}$ denotes the nearest neighbor in the graphene lattice.

how the $\mathrm{Cl}-\mathrm{Cl}$ interaction changes with their separation. The $\mathrm{Cl}-\mathrm{Cl}$ coupling is practically negligible when their separation is greater or equal to $3.6 \AA$ corresponding to the fourth nearest neighbor. For smaller separations corresponding to the third nearest neighbor separation of graphene with the threshold distance, they form the $\mathrm{Cl}_{2}$ molecule and desorb from the graphene surface, since the $\mathrm{Cl}-\mathrm{Cl}$ interaction energy becomes stronger than the sum of the binding energies of two $\mathrm{Cl}$ atoms to graphene $(2.20 \mathrm{eV})$. Accordingly, the gain of energy through the formation of $\mathrm{Cl}_{2}$ is $1.26 \mathrm{eV}$ per molecule. This explains also why chlorination of graphene is endothermic. There is a weak $\mathrm{vdW}$ interaction between bare graphene and the $\mathrm{Cl}_{2}$ molecule. The maximum interaction of $144 \mathrm{meV}$ occurs when the molecule is parallel to a $\mathrm{C}-\mathrm{C}$ bond and $3 \AA$ above it.

Next we examine how the electronic structure changes with one-sided uniform coverage. In Figure 3 we show the band structures calculated for a single $\mathrm{Cl}$ atom adsorbed to th $\mathrm{e}(n \times$ $n$ ) supercell for $n=2,3,5$, and 6 . Here $n=2$ and 3 correspond to $\Theta=0.125$ and 0.056 . The phonon calculations for these two coverage values are found to be stable, since the frequencies of phonon modes are positive for any $\mathbf{k}$-point in BZ. The smallest separation between $\mathrm{Cl}$ atoms is larger than the threshold distance of $2.8 \AA$ (i.e., the third nearest neighbor distance) even for $\Theta=0.125$. We see that with increasing $\Theta$ the dispersion of $\mathrm{Cl}$ bands increases slightly and the linearly crossing bands start to split. The magnetic moment also increases with coverage, since the splitting of spin-up and spin-down bands increases. We found $\mu=0.64 \mu_{\mathrm{B}}$ and $0.56 \mu_{\mathrm{B}}$ for $\Theta=0.0555$ (or $n=3$ ) and 0.020 (or $n=5$ ), respectively. On the other hand, a single

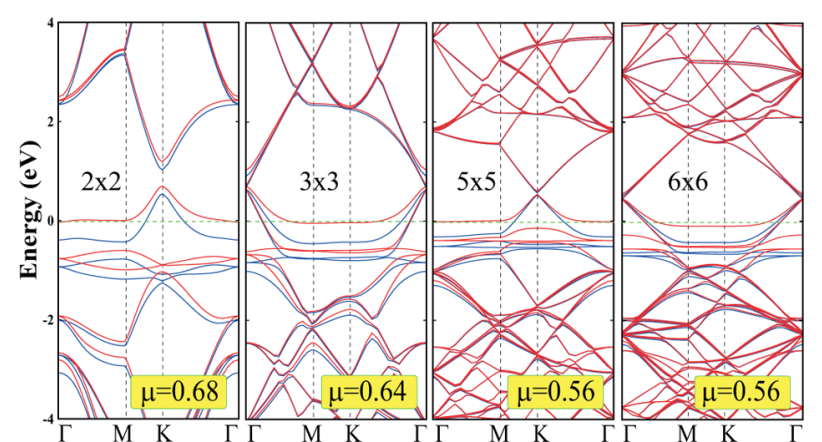

Figure 3. Energy band structure of a single $\mathrm{Cl}$ atom adsorbed to each $(n \times n)$ supercell of graphene for $n=2,3,5$, and 6, which correspond to the one-sided uniform coverage $\Theta=1 / 2 n^{2}$. For $n \geq 2$ the distance between nearest $\mathrm{Cl}$ adatoms is larger than the threshold distance described in Figure 2. Whereas stable $\mathrm{Cl}$ coverage with $n=1$ (or $\Theta=$ 0.5 ) cannot be achieved due to the strong $\mathrm{Cl}-\mathrm{Cl}$ coupling. The unit of magnetic moment, $\mu$, is Bohr magneton per $(n \times n)$ supercell. The zero of energy is set at the Fermi level, $E_{\mathrm{F}}$.

$\mathrm{Cl}$ adsorption to each $(1 \times 1)$ unit cell of graphene (which corresponds to $\Theta=0.5$ ) is unstable due to a $\mathrm{Cl}-\mathrm{Cl}$ distance smaller than $2.8 \AA$. Since the uniformly chlorinated graphene is found to be metallic for any coverage studied here, the experimental studies measuring a band gap of $\sim 0.05 \mathrm{eV}$ should correspond to two-sided coverage.

To understand the formation of chlorinated domains on graphene, and hence to analyze the kinetics of $\mathrm{Cl}$ coverage, we next examine the adsorption of a second $\mathrm{Cl}$ atom at different sites in close proximity to the first one in a $(4 \times 4)$ supercell. When a $\mathrm{Cl}$ is placed on top of a carbon atom, the second $\mathrm{Cl}$ can be adsorbed on six relevant sites on the hexagonal carbon ring as shown in Figure 4a.

Among the various possible cases, the ortho top-bottom arrangement is the most favorable one. While adsorption of a single $\mathrm{Cl}$ atom yields $0.05 \AA$ buckling of the graphene lattice, adjacent $\mathrm{C}$ atoms forming $\mathrm{C}-\mathrm{Cl}$ bonds in ortho top-bottom configuration are buckled by $0.46 \AA$. In Figure $4 \mathrm{~b}$ the contour plots of total charge density on the planes passing through the bonds clearly explain the ionic character of the $\mathrm{C}-\mathrm{Cl}$ bond of a single $\mathrm{Cl}$ adatom. In the case of ortho top-bottom the bonding between the $\mathrm{Cl}$ adatom and the $\mathrm{C}$ atom of graphene has $\mathrm{sp}^{3}$ type covalent character. While the charge density between $\mathrm{Cl}$ and $\mathrm{C}$ atoms is very low in the ionic case, the bond charge is enhanced in the covalent $\mathrm{C}-\mathrm{Cl}$ bond. The binding energy of the covalent bond is stronger $\left(E_{\mathrm{b}}=1.53 \mathrm{eV}\right)$ and consequently its length is shorter $(d=1.90 \AA$, which is comparable to the empirical covalent radii of the $\mathrm{C}-\mathrm{Cl}$ bond or the bond length $d_{\mathrm{C}-\mathrm{Cl}}=1.77 \AA$ of the $\mathrm{CCl}_{4}$ molecule). The binding energies per bond in the case of meta and para top-bottom configurations are $E_{\mathrm{b}}=1.10$ and $1.31 \mathrm{eV}$, respectively. In spite of the fact that the ortho top-bottom configuration is $0.52 \mathrm{eV}$ less energetic than the formation of the $\mathrm{Cl}_{2}$ molecule, it can be stable since the formation of the $\mathrm{Cl}_{2}$ molecule from two $\mathrm{Cl}$ atoms at different sides is hindered. In para and meta top-bottom structures, the binding energies of two bonds are further lowered from the formation energy of the $\mathrm{Cl}_{2}$ molecule.

The situation is however dramatically different for one-sided (top-top) configurations presented in the second row of Figure 4a. For these three one-sided configuration, namely, ortho, para, and meta top-top configurations, the $\mathrm{Cl}-\mathrm{Cl}$ distances are within the third nearest neighbors. Owing to the strong $\mathrm{Cl}-\mathrm{Cl}$ 
(a)
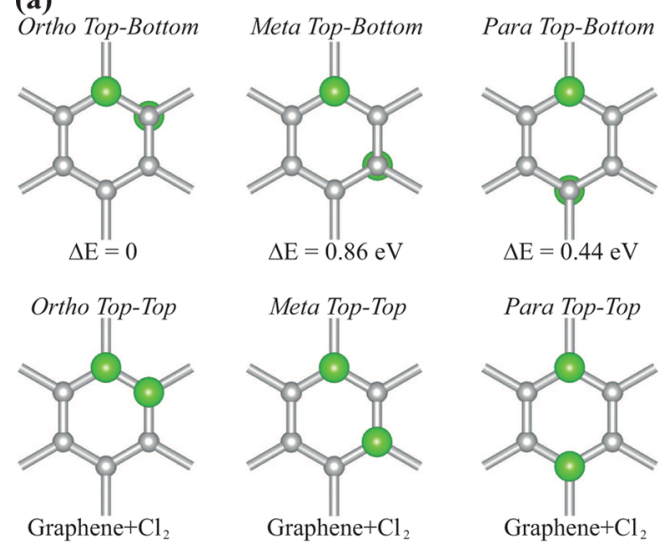

(b) Graphene + Chlorine

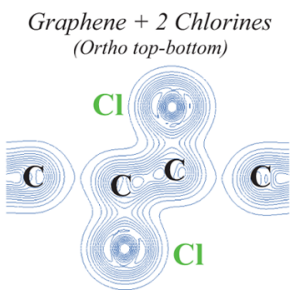

Figure 4. (a) The atomic structure of two $\mathrm{Cl}$ atoms adsorbed to a $(4 \times$ 4) supercell of graphene. In the three different configurations illustrated in the top panels, namely ortho top-bottom, para topbottom, and meta top-bottom, two adsorbed $\mathrm{Cl}$ atoms are stable. $\Delta E$ indicates their energies relative to the total energy of the ortho topbottom configuration. Double sided adsorption imposes a local buckling in the planar graphene. Three one-sided configurations, ortho top-top, para top- top, and meta top-top are not allowed; $\mathrm{Cl}$ atoms cannot be bound to graphene, they rather form a $\mathrm{Cl}_{2}$ molecule. Large green and small gay balls represent $\mathrm{Cl}$ and $\mathrm{C}$ atoms, respectively. (b) Contour plots of the total charge density of a single $\mathrm{Cl}-\mathrm{C}$ bond and two $\mathrm{Cl}-\mathrm{C}$ bonds in the ortho top-bottom configuration. Contour spacings between $0.025 e / \AA^{3}$ and $1.0 e / \AA^{3}$ are $0.025 e / \AA^{3}$.

coupling, the formation of the $\mathrm{Cl}_{2}$ molecule is energetically favored. Accordingly, unlike the half-fluorinated graphene ${ }^{23}$ $\left(\mathrm{C}_{2} \mathrm{~F}\right)$, one-sided densely chlorinated graphene with the coverage $\Theta=0.5$, namely, $\mathrm{C}_{2} \mathrm{Cl}$, cannot be realized.

We now concentrate on the chemical conversion of graphene to a fully chlorinated graphene structure $(\Theta=1.0)$, called chlorographene. In addition to well-known boat, chair, and nonbonding chair conformations one can also consider zigzag and armchair stoichiometric chlorographene configurations shown in Figure 5. Here, boat, chair, nonbonding chair, and zigzag conformations are treated by using a $(2 \times 2)$ supercell; a $(4 \times 4)$ supercell is required for the armchair conformations. As a consequence of the strong $\mathrm{Cl}-\mathrm{Cl}$ interaction, boat, zigzag, and armchair configurations of $\mathrm{Cl}$ atoms cannot remain stable on graphene.

Here the nonbonding chair conformations in Figure $5 b$ consisting of a planar graphene layer sandwiched between two $\mathrm{Cl}$ layers deserve detailed discussion. In this conformation $\mathrm{Cl}$ atoms are placed to the alternating $\mathrm{T}$-sites at both sides of graphene. Earlier theoretical studies ${ }^{30,31}$ have predicted that this metallic structure has the highest cohesive energy among conformations described in Figure 5. Present calculations predict $E_{\mathrm{T}}=23.35 \mathrm{eV} /$ per primitive cell and cohesive energy $E_{\text {coh }}=2.79 \mathrm{eV} /$ per primitive cell. Even if the structure optimization using the conjugate gradient method performed in the $(1 \times 1)$ hexagonal unit cell finds the nonbonding chair

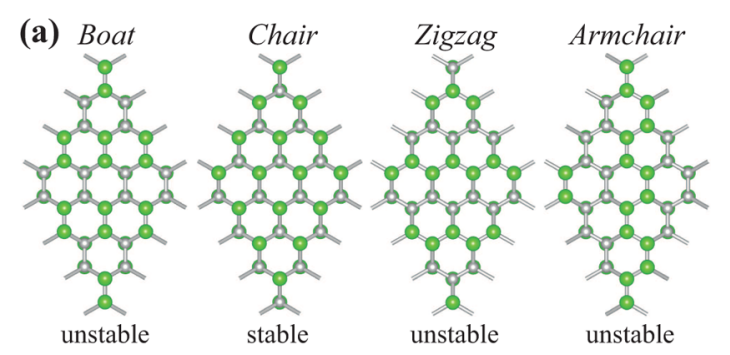

(b) Nonbonding Chair

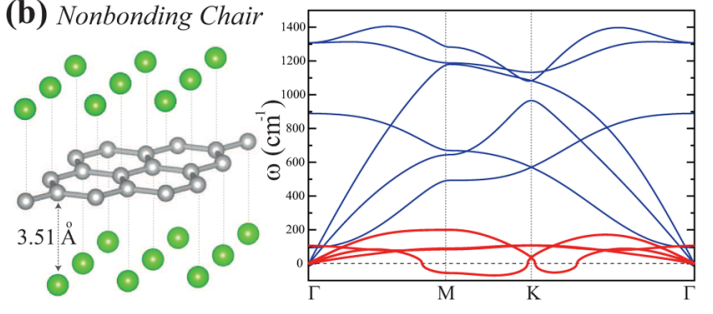

Figure 5. (a) Top view of atomic structures of boat, chair (i.e., the covalently bonded and buckled graphene layer is sandwiched between two planar $\mathrm{Cl}$ layers), zigzag, and armchair conformations. Green and small gay balls represent $\mathrm{Cl}$ and $\mathrm{C}$ atoms, respectively. (b) Side view of the nonbonding chair conformation consisting of one planar graphene layer sandwiched by two planar $\mathrm{Cl}$ layers and its calculated phonon dispersion curves. Low-frequency phonon modes shown by red lines are related to adsorbed $\mathrm{Cl}$ atoms. These modes have imaginary frequencies and hence they are unstable.

structure stable, we carried out an extensive analysis of this conformation. We found that the attractive interaction between each $\mathrm{Cl}$ layer and underlying graphene is rather small $(50 \mathrm{meV}$ per unit cell) in spite of the fact that the graphene lattice expanded by $2.8 \%$ and the system attained a total energy $\sim 3.31$ $\mathrm{eV}$ higher than that of graphene. The weak interaction between $\mathrm{Cl}$ and $\mathrm{C}$ atoms explains why the distance from the $\mathrm{Cl}$ layer to graphene is large (3.51 $\AA$ ). This large distance and minute charge transfer from carbon to the $\mathrm{Cl}$ atom can be contrasted with the $\mathrm{C}-\mathrm{Cl}$ distance $(2.54 \AA)$ and the transfer of 0.44 electrons from $\mathrm{C}$ to $\mathrm{Cl}$ of the ionic bond of the single $\mathrm{Cl}$ atom adsorbed to graphene as discussed in Section III. This paradoxical situation occurred due to the strong intralayer coupling among $\mathrm{Cl}$ atoms in both $\mathrm{Cl}$ layers at both sides of graphene. In fact, the binding energy of $\mathrm{Cl}$ atoms of a single $\mathrm{Cl}$ layer having the same atomic configuration as the $\mathrm{Cl}$ layers of the nonbonding chair conformation is found to be $1.41 \mathrm{eV}$ per $\mathrm{Cl}$ atom. It appears that high cohesive energy of nonbonding chair conformation relative to free graphene and free $\mathrm{Cl}$ atoms is attained mainly by the cohesion between $\mathrm{Cl}$ atoms, but not by the interaction between $\mathrm{Cl}$ and carbon atoms. In addition, GGA+vdW calculations performed for nonbonding conformation resulted in optimized structure close to that obtained by using LDA.

Under these paradoxical situations we further examined the stability of the metallic nonbonding chair conformer by carrying out $\mathrm{ab}$ initio calculations of phonon frequencies in the BZ. As seen in Figure 5b, the calculated phonon branches, which are associated with $\mathrm{Cl}$ in-plane and out-of-plane modes and hence are practically isolated from graphene modes, have imaginary frequencies near $M$ - and $K$-high symmetry points. This clearly indicates that the nonbonding chair structure is unstable at $T=0 \mathrm{~K}$. In fact, a minute displacement or perturbation of atoms in the $\mathrm{Cl}$ plane results in the breakdown of Cl-layers, if the nonbonding chair structure is treated by 
using a $(4 \times 4)$ supercell. It appears that structure optimization in earlier studies ${ }^{30,31}$ carried out in a single cell has limited the degree of freedom of $\mathrm{Cl}$ atoms preventing them from reconstructions involving large displacements or from forming molecules. Accordingly, the stability imposed by the structure optimization using primitive cell was unrealistic. Even though the nonbonding chair conformation has the lowest energy among other conformers including (covalently bonded) the chair structure in Figure 5, it corresponds either to a very shallow local minimum or to a saddle point for specific directions of atomic displacements in the Born-Oppenheimer surface.

\section{STABLE FULLY CHLORINATED GRAPHENE: CHLOROGRAPHENE}

A. Structural Properties. In contrast to nonbonding chair conformation, our analysis indicates that like graphane $(\mathrm{CH})$ and fluorographene (CF), the chair structure, where one chlorine atom is attached to each carbon atom of the buckled graphene alternatingly from top and bottom sides, can be a stable structure. The optimized atomic structure and the hexagonal lattice are shown in Figure 6a. The present study

(a)

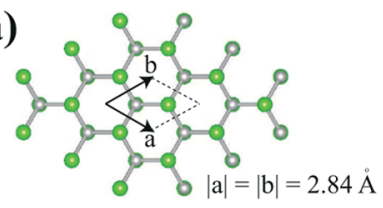

(b)
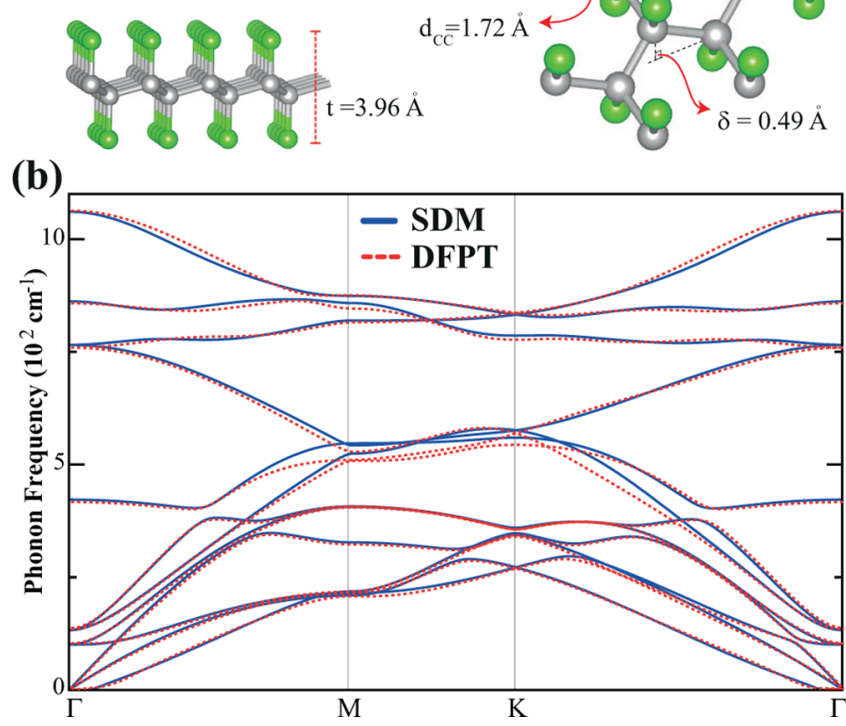

Figure 6. (a) Top, side, and tilted views for the atomic structure of the chlorographene $\mathrm{CCl}$ layer having hexagonal lattice and honeycomb structure. Carbon and chlorine atoms are indicated by gray (dark) and green (light) balls, respectively. Calculated structural parameters are indicated. (b) Phonon bands of chlorographene calculated by using by $\mathrm{SDM}^{43}$ and $\mathrm{DFPT}^{44}$ methods.

predicts its total energy $E_{\mathrm{T}}=22.24 \mathrm{eV} /$ per primitive cell and its cohesive energy $E_{\text {coh }}=1.68 \mathrm{eV}$. In the rest of this paper we call this stable structure chlorographene or $\mathrm{CCl}$, which should be distinguished from the unstable, nonbonding chair structure presented in the previous section in Figure $5 \mathrm{~b}$. The remarkable situation is that the $\mathrm{sp}^{2}$-bonded planar graphene is buckled by $\delta$ $=0.50 \AA$, once it is converted to CCl. This way the local configuration of three-folded $\mathrm{sp}^{2}$-bonding has changed to four- folded $\mathrm{sp}^{3}$-bonding, reminiscent of tetrahedrally coordinated diamond structures. Formation of four-folded $\mathrm{sp}^{3}$-bonding through buckling is essential for the stabilization of $\mathrm{CCl}$, despite the $\mathrm{C}-\mathrm{C}$ distance increased from $1.42 \AA$ to $1.72 \AA$. On the other hand, while the $\mathrm{C}-\mathrm{Cl}$ ionic bond in the adsorption of a single $\mathrm{Cl}$ adatom is $2.54 \AA$ in Section III, it transforms to a covalent bond by contracting to $1.73 \AA$ in chlorographene, which is also very close to the bond length of the $\mathrm{CCl}_{4}$ molecule. The bond length of chlorographene can also be contrasted with the $\mathrm{Cl}$-graphene distance in the nonbonding chair structure discussed in the previous section. We note that chlorographene is stable despite its cohesive energy being smaller than that of the unstable nonbonding chair conformation. This is due the fact that chlorographene corresponds to a local minimum in the Born-Oppenheimer surface.

In Table 1 we compare the structural parameters, relevant energies like cohesive energy, formation energy, and desorption energy of graphene, $\mathrm{CH}, \mathrm{CF}$, and $\mathrm{CCl}$, all calculated by using LDA. It is seen that chlorographene, $\mathrm{CCl}$ has a binding energy $E_{\mathrm{b}}=1.68 \mathrm{eV}$ per unit cell relative to bare graphene and the free $\mathrm{Cl}$ atom, and lowest $E_{\text {coh }}(19.60 \mathrm{eV})$ per unit cell among other possible graphene derivatives. However, once $\mathrm{CCl}$ is synthesized a desorption energy of $E_{\mathrm{des}}=1.28 \mathrm{eV}$ is required to take a single $\mathrm{Cl}$ atom out.

B. Vibrational Properties and Raman Spectra. Chlorographene $(\mathrm{CCl})$ possesses $D_{3 d}$ point group symmetry. Phonon bands of $\mathrm{CCl}$ are shown in Figure $6 \mathrm{~b}$. It is seen that all the phonon modes have positive frequencies and hence the predicted structure of chlorographene is stable at $T=0 \mathrm{~K}$. Unlike the phonon bands of the nonbonding conformer, $\mathrm{C}-\mathrm{Cl}$ bonds of chlorographene participate in all acoustic phonon branches. Therefore, the phonon spectrum of chlorographene differs from the spectrum of graphene. Though the chlorographene belongs to the same space group as $\mathrm{CH}$ and $\mathrm{CF}{ }^{22,23}$ the phonon frequencies are lowered (softened) due to the saturation of $\mathrm{C}$ atoms with heavy $\mathrm{Cl}$ atoms. While $\mathrm{LA}$ and TA modes are linearly dependent on the phonon wave vector, the ZA mode has a quadratic dispersion in the vicinity of the $\Gamma$ point. $^{22,23,52}$ As compared to a single layer graphene, LO and TO (ZO) optical modes are softened from 1600 (900) to 1061 (421) $\mathrm{cm}^{-1}$ due the existence of surrounding $\mathrm{Cl}$ layers. In general, both phonon bands calculated by using SDM and DFPT agree well, but they differ slightly at $\mathrm{M}$ - and K-points for optical phonon branches near $500 \mathrm{~cm}^{-1}$.

Group theory analysis shows that the decomposition of the vibration representation at the $\Gamma$-point is $\Gamma=2 A_{1 \mathrm{~g}}+2 A_{2 \mathrm{u}}+4 E_{\mathrm{g}}$ $+4 E_{\mathrm{u}}$. Among these, the modes at 105, 398, 715, and 1042 $\mathrm{cm}^{-1}$ are bond stretching modes and are Raman-active. Raman mode $A_{1 \mathrm{~g}}$ at 1042 (398) is entirely due to the out-of-plane vibration of $\mathrm{C}$ and $\mathrm{Cl}$ atoms moving in the same (opposite) direction with respect to each other. The observation of these Raman active modes is expected to shed light on the $\mathrm{Cl}$ coverage and the structure of chlorinated graphene. The observation of the characteristic D-peak at 1330, the G-peak at 1587 , and the $2 \mathrm{D}$-peak at $2654 \mathrm{~cm}^{-1}$ from chlorinated graphene indicates low coverage of $\mathrm{Cl}^{26}$ Since the Raman measurements were performed in the range of $1250-3500 \mathrm{~cm}^{-1}$, possible Raman-active peaks originating from chlorine atoms could not be observed.

C. Stability of $\mathrm{CCl}$ at Finite Temperature. Here we investigate whether $\mathrm{CCl}$ is stable at finite temperature, even though all phonon modes having positive frequencies in the Brillouin zone indicate its stability at $T=0 \mathrm{~K}$. This is achieved 
Table 1. Calculated Values for Graphene, and Graphene Derivatives, Such As Graphane CH, Fluorographene CF, and Chlorographene $\mathrm{CCl}^{a}$

\begin{tabular}{|c|c|c|c|c|c|c|c|c|c|c|c|c|c|}
\hline material $(\mathrm{CX})$ & $a, \AA$ & $d_{\mathrm{CC}}, \AA$ & $d_{\mathrm{CX}}, \AA$ & $t, \AA$ & $\Phi, \mathrm{eV}$ & $\Delta \rho, e$ & $E_{\text {coh }}, \mathrm{eV}$ & $E_{\mathrm{f}}, \mathrm{eV}$ & $E_{\mathrm{des}}^{\mathrm{X}}, \mathrm{eV}$ & $E_{\mathrm{g}}, \mathrm{eV}$ & $E_{\mathrm{g}}^{G W_{0}}, \mathrm{eV}$ & $C, \mathrm{~J} / \mathrm{m}^{2}$ & R-active modes, $\mathrm{cm}^{-1}$ \\
\hline graphene & 2.46 & 1.42 & & & 4.49 & & 17.87 & & & & & 335 & 1600 \\
\hline $\mathrm{CH}$ & 2.51 & 1.52 & 1.12 & 2.68 & 4.79 & 0.06 & 23.60 & +0.39 & 4.79 & 3.42 & 5.97 & 243 & $1162,1164,1341,2806$ \\
\hline $\mathrm{CF}$ & 2.55 & 1.55 & 1.37 & 3.22 & 7.93 & -0.61 & 25.31 & +2.04 & 5.46 & 2.96 & 7.49 & 250 & $245,681,1264,1305$ \\
\hline $\mathrm{CCl}$ & 2.84 & 1.72 & 1.73 & 3.96 & 3.67 & -0.13 & 19.60 & -0.95 & 1.28 & 1.21 & 4.33 & 186 & $105,398,715,1042$ \\
\hline
\end{tabular}

${ }^{a}$ The calculated values are lattice constant $(a)$; C-C bond distance $\left(d_{\mathrm{CC}}\right)$; C-X bond distance $\left(d_{\mathrm{CX}}\right)$; thickness of the layer as described in Figure $6 \mathrm{a}$ $(t)$; photoelectric threshold $(\Phi)$; charge transfer from $\mathrm{C}$ to $\mathrm{X}(\Delta \rho)$; cohesive energy per unit cell $\left(E_{\text {coh }}\right)$, formation energy $\left(E_{\mathrm{f}}\right)$, desorption energy of a single $\mathrm{X}$ atom from the $\mathrm{CX}$ surface $\left(E_{\text {des }}\right)$; direct band gap $\left(E_{\mathrm{g}}\right)$; band gap corrected with $\mathrm{GW}_{\mathrm{o}}, E_{\mathrm{g}}^{G W_{0}}$; and in-plane stiffness $(C)$; Raman active modes. Energies are calculated in $(2 \times 2)$ supercell.

by carrying out $\mathrm{ab}$ initio molecular dynamics (MD) calculations at finite temperatures, using a $(6 \times 6)$ supercell involving 144 atoms. Here we summarize our findings. At relatively low temperatures, for example, at $T=500 \mathrm{~K}$, the perfect $\mathrm{CCl}$ remained stable even after $\sim 6000$ time steps of 2 fs. Even if 6000 time steps is large for ab initio calculations, but low to attain reliable statistics, this result suggests the stability of perfect $\mathrm{CCl}$ near room temperature. However, $\mathrm{Cl}$ atoms dissociate from $\mathrm{CCl}$ at $1000 \mathrm{~K}$. This may be interpreted that $\mathrm{CCl}$ cannot be stable at elevated temperatures. $\mathrm{Ab}$ initio $\mathrm{MD}$ calculations with a single $\mathrm{Cl}$ vacancy at one side brought about the formation of a second vacancy at the other side after 200 time steps at $500 \mathrm{~K}$. Thereafter, the structure continued to be stable. However, an island of $\mathrm{Cl}$ at both sides of graphene, for example, $6 \mathrm{Cl}$ atoms adsorbed to a hexagon of graphene alternatingly from the top and bottom side of graphene, is found unstable.

Our analysis using finite temperature ab initio $\mathrm{MD}$ calculations led us to draw the following conclusion from the above results: A perfect $\mathrm{CCl}$ is stable at $T=0 \mathrm{~K}$ and can remain stable possibly at room temperature. The creation of a single vacancy at one site imposes the formation of a second vacancy at the opposite side. This pair of vacancies can survive at room temperature. However, $\mathrm{CCl}$ having vacancies or holes is vulnerable to dissociation through the formation of $\mathrm{Cl}_{2}$ molecules. The negative formation energy underlies these instabilities.

D. Electronic Properties. We present the band structure and the orbital decomposed density of states of fully chlorinated graphene $\mathrm{CCl}$ in Figure 7. As a consequence of transition from $\mathrm{sp}^{2}$ - to of $\mathrm{sp}^{3}$-type bonding and the mixing with $\mathrm{Cl}$ orbitals through $\mathrm{Cl}-\mathrm{C}$ covalent bonds, linearly crossing semimetallic bands of graphene changes dramatically and turn to a nonmagnetic semiconductor with a direct band gap of $E_{\mathrm{g}}=$ $1.21 \mathrm{eV}$ (GGA+vdW value: $1.55 \mathrm{eV}$ ) at the center of BZ. The 2 -fold degenerate bands at the top of the valence band are mainly composed of the $\mathrm{p}_{x y}$ valence orbitals of $\mathrm{Cl}$ and $\mathrm{C}$ atoms and belong to the $E_{\mathrm{g}}$ irreducible representation. However, the nondegenerate conduction band edge is formed by the hybridization of $\mathrm{C}-\mathrm{p}_{x y}, \mathrm{C}-\mathrm{p}_{z}, \mathrm{C}-\mathrm{s}$, and $\mathrm{Cl}-\mathrm{p}_{z}$ and belongs to the $A_{2 u}$ irreducible representation. Calculated values relevant for the electronic properties of $\mathrm{CCl}$ are given together with those of graphene, $\mathrm{CH}$, and $\mathrm{CF}$ in Table 1 . $\mathrm{CCl}$ has the lowest band gap $(1.21 \mathrm{eV})$ among graphene derivatives, i.e. graphane $\left(E_{g}=3.42 \mathrm{eV}\right)$ and fluorographene $\left(E_{\mathrm{g}}=2.96 \mathrm{eV}\right)$. Our calculations also reveals that the experimentally observed energy gap of $0.045 \mathrm{eV}$ may arise from the partially chlorinated regions. Since DFT usually underestimates the band gaps, the band gap of $3 \mathrm{D}$ bulk crystals is successfully corrected by the $G W_{0}$ self-energy method. In the present paper we apply the

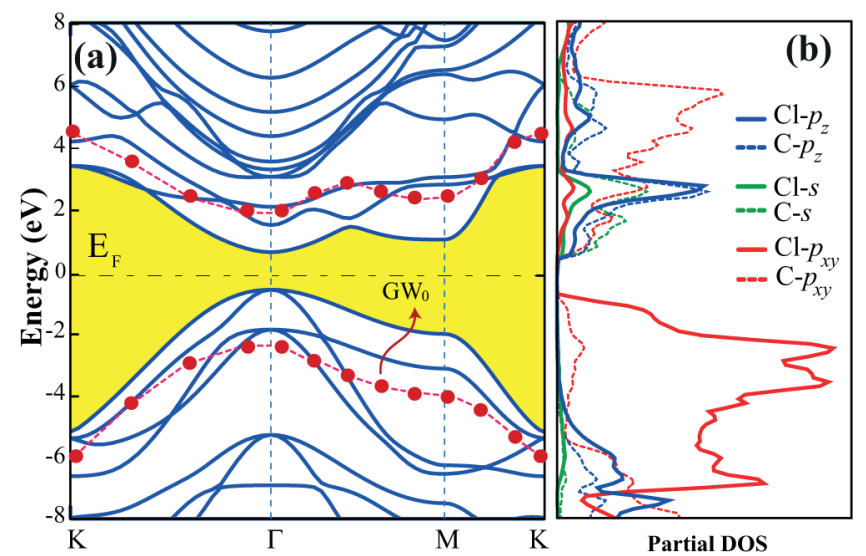

Figure 7. (a) Electronic band structure of chlorographene $\mathrm{CCl}$. The band gap is shaded yellow. The $\mathrm{GW}_{\mathrm{o}}$ corrected valence and conduction bands are shown by a dashed line and red balls. The zero of energy is set to the Fermi level $E_{\mathrm{F}}$. (b) Density of states projected to various orbitals (PDOS).

$G W_{0}$ method to correct valence and conduction bands and hence the band gap of $\mathrm{CCl}$. We found the corrected band gap to be $4.33 \mathrm{eV}$. This is a dramatic increase. A similar situation occurred for the correction of band gaps carried out for single layer structures, such as $\mathrm{BN},{ }^{53} \mathrm{CF},{ }^{23}$ and $\mathrm{MoS}_{2} .{ }^{54}$ In particular, while the LDA predicted band gap of a single layer $\mathrm{MoS}_{2}$ agrees with experiment, the $G W_{0}$ correction yielded a very large band gap. This situation led us to question whether the $G W_{0}$ correction is suitable for single layer structures.

While graphane has a positively charged surface due to the electron transferred from $\mathrm{H}$ to $\mathrm{C}$ atoms, $\mathrm{Cl}$ layers of $\mathrm{CCl}$ are negatively charged since 0.13 electrons (GGA+vdW value: 0.10 electrons) are transferred from $\mathrm{C}$ to $\mathrm{Cl}$ atoms. We also note that the effective charge is reduced from 0.42 to 0.13 electrons by going from the ionic $\mathrm{C}-\mathrm{Cl}$ bond in single $\mathrm{Cl}$ adsorption to the covalent $\mathrm{C}-\mathrm{Cl}$ bond of chlorographene. Since $\mathrm{CCl}$ surfaces are negatively charged, it is possible to lower the photoelectric threshold of graphene from 4.49 to $3.67 \mathrm{eV}$ by covering its surfaces with chlorine atoms. In contrast, as one notices in Table 1, both fluorinated ${ }^{23}$ and hydrogenated ${ }^{14,22}$ derivatives of graphene have photoelectric thresholds higher than graphene and $\mathrm{CCl}$. The lower photoelectric threshold provides materials highly emissive surfaces. This facile photoemission feature is desirable for fast laser applications. ${ }^{55,56}$

E. Mechanical Properties. Earlier studies have shown that graphane $^{15,16}$ and fluorographene $\mathrm{e}^{22,23}$ derivatives are strong materials like graphene. Among the three graphene derivatives chlorographene is the thickest one and we can expect some different mechanical properties. We will discuss the structural rigidity of chlorographene within the harmonic range of the 
elastic deformation, where the structure responded to strain $\epsilon$ linearly and reversibly. In the elastic range, in-plane stiffness $C$ would be a good measure of the response of material. Here we use the expression to calculate in-plane stiffness, $C=(1 /$ $\left.A_{0}\right) \cdot\left(\mathrm{d}^{2} E_{\mathrm{s}} / \mathrm{d} \epsilon^{2}\right)$, where $A_{0}$ is the equilibrium area of the supercell and the strain energy is defined as the total energy at a given uniform strain minus the total energy at zero strain, namely, $E_{\mathrm{s}}$ $=E_{\mathrm{T}}(\epsilon)-E_{\mathrm{T}}(\epsilon=0)$. Here the strain in one direction is $\epsilon=\Delta c /$ $c_{0}$, where $c_{0}$ is the equilibrium lattice constant of the supercell and $\Delta c$ is its stretching. For $\mathrm{CCl}$ we calculated $C$ as $186 \mathrm{~J} / \mathrm{m}^{2}$, which identifies it as strong as $\mathrm{CH}$ and $\mathrm{CF}$.

In Figure 8a we show the variation of the strain energy $E_{\mathrm{s}}$ and its derivative with respect to the applied uniform strain, $\mathrm{d} E_{\mathrm{s}} / \mathrm{d} \epsilon$.
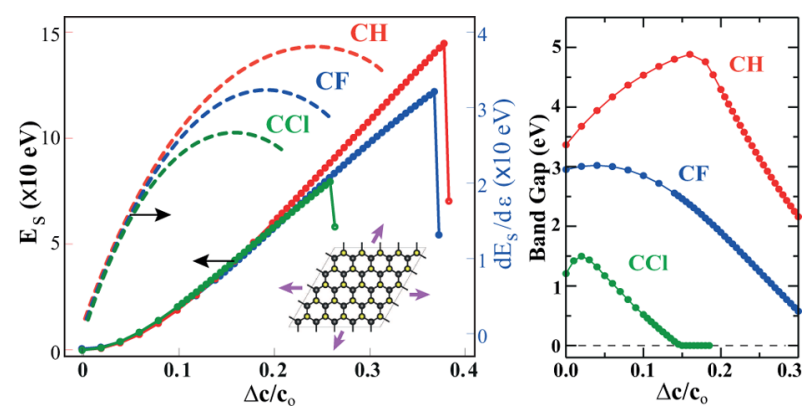

Figure 8. (a) The variation of the strain energy $E_{\mathrm{s}}$ (curves on the righthand side) and its derivative with respect to applied uniform strain $\epsilon$, i.e., $\mathrm{d} E_{\mathrm{s}} / \mathrm{d} \epsilon$ (curves on the left-hand side), are calculated for $\mathrm{CCl}, \mathrm{CH}$, and CF. After the maxima these structures become unstable and undergo a plastic deformation. (b) Variation of the band gap with uniform strain. Calculations performed in $(5 \times 5)$ supercells.

The latter is linear for small $\epsilon$ in the harmonic range. The elastic deformation occurs until the maximum of $\mathrm{d} E_{\mathrm{s}} / \mathrm{d} \epsilon$, whereby the structure attains its initial state when the applied strain is lifted. Beyond the maximum the structural instability sets in with irreversible deformations. The region beyond the maximum is called the plastic region. It is seen that $\mathrm{CCl}$ undergoes instability, under 0.15 expansion of the lattice, whereas the critical expansion values are 0.20 and 0.24 for $\mathrm{CF}$ and $\mathrm{CH}$, respectively.

We also calculated the effect of the elastic strain on the band gap of $\mathrm{CCl}, \mathrm{CF}$, and $\mathrm{CH}$ and presented our results in Figure $8 \mathrm{~b}$. $\mathrm{CH}$ and $\mathrm{CCl}$ have similar response to elastic strain: The increase of their band gaps for small strain is followed by a rapid decrease for large strain. In contrast, initially the band gap of CF does not show any significant increase with increasing strain; for small strain it is almost unaltered, but decreases rapidly for large strain. Because of its smaller band gap, the metalization of $\mathrm{CCl}$ occurs earlier in the course of expansion.

\section{CONCLUSIONS}

Motivated by the recent work of $\mathrm{Li}$ et al., ${ }^{26}$ who achieved the photochemical chlorination of a bare graphene, we performed a first-principle study on the chlorination of graphene starting from a single $\mathrm{Cl}$ adatom adsorption to fully chlorinated graphene $\mathrm{CCl}$. We found that even if a $\mathrm{Cl}$ atom can be bound to graphene with a significant binding energy, it can migrate on the surface of graphene almost without an energy barrier. Formation of a $\mathrm{Cl}_{2}$ molecule from two individual, migrating adatoms is energetically favorable when they are at close proximity. Therefore the formation energy of the adsorption of a single $\mathrm{Cl}$ atom relative to a $\mathrm{Cl}_{2}$ molecule is negative. In this respect, all possible configurations or decoration obtained by the chlorination of one surface of graphene and considered in this study are not stable. On the other hand, the configuration where two $\mathrm{Cl}$ atoms are adsorbed from opposite sides of graphene to two adjacent carbon atoms forming $\mathrm{sp}^{3}$-type covalent $\mathrm{C}-\mathrm{Cl}$ bonds and hence inducing buckling is stable, since the dissociation of these two $\mathrm{Cl}$ atoms by forming a $\mathrm{Cl}_{2}$ molecule is hindered. Once the bare graphene is fully chlorinated from both sides inducing buckling of carbon atoms, the resulting conformation, called chlorographene, is stable at $T=0 \mathrm{~K}$ and also possibly at room temperature. With its $1.21 \mathrm{eV}$ direct band gap, stiff mechanical properties, and response to homogeneous strain this material displays interesting properties for future technological applications. On the other hand, the nonbonding chair conformation has cohesive energy higher than that of chlorographene in the chair conformation and is found to be unstable. While both structures are not in the ground state, chlorographene corresponds to a local minimum on the Born-Oppenheimer surface. Of course, a state having energy lower than those of both conformation is $\mathrm{Cl}_{2}$ molecules, which are weakly attached to bare graphene.

\section{AUTHOR INFORMATION}

\section{Corresponding Author}

* E-mail: ciraci@fen.bilkent.edu.tr.

\section{Present Address}

${ }^{\perp}$ Departement Fysica, Universiteit Antwerpen, Groenenborgerlaan 171, B-2020 Antwerpen, Belgium

\section{Notes}

The authors declare no competing financial interest.

\section{ACKNOWLEDGMENTS}

The authors thank Dr. M. Topsakal for his assistance regarding the stability of chlorographene and Mr. O. Ozcelik for his assistance in specific calculations. This work is supported by TUBITAK through Grant No. 108T234. Part of the computational resources was provided by TUBITAK ULAKBIM, High Performance and Grid Computing Center (TR-Grid eInfrastructure). S.C. acknowledges the partial support of TUBA, Academy of Science of Turkey.

\section{REFERENCES}

(1) Novoselov, K.; Geim, A.; Morozov, S.; Jiang, D.; Zhang, Y.; Dubonos, S.; Grigorieva, I.; Firsov, A. Science 2004, 306, 666-669.

(2) Novoselov, K. S.; Geim, A. K.; Morozov, S. V.; Jiang, D.; Katsnelson, M. I.; Grigorieva, I. V.; Dubonos, S. V.; Firsov, A. A. Nature 2005, 438, 197-200.

(3) Geim, A. K.; Novoselov, K. S. Nat. Mater. 2007, 6, 183-191.

(4) Geim, A. K. Science 2009, 324, 1530-1534.

(5) Katsnelson, M. I.; Novoselov, K. S.; Geim, A. K. Nat. Phys. 2006, 2, 620-625.

(6) Dikin, D. A.; Stankovich, S.; Zimney, E. J.; Piner, R.; Dommett, G. H. B.; Evmenenko, G.; Nguyen, S. T.; Ruoff, R. S. Nature 2007, 448, 457-460.

(7) Eda, G.; Fanchini, G.; Chhowalla, M. Nat. Nanotechnol. 2008, 3, 270-274.

(8) Gomez-Navarro, C.; Weitz, R. T.; Bittner, A. M.; Scolari, M.; Mews, A.; Burghard, M.; Kern, K. Nano Lett. 2007, 7, 3499-3503.

(9) Gilje, S.; Han, S.; Wang, M.; Wang, K. L.; Kaner, R. B. Nano Lett. 2007, 7, 3394-3398.

(10) Robinson, J. T.; Perkins, F. K.; Snow, E. S.; Wei, Z.; Sheehan, P. E. Nano. Lett. 2008, 8, 3137-3140. 
(11) Kim, S.; Zhou, S.; Hu, Y.; Acik, M.; Chabal, Y. J.; Berger, C.; de

Heer, W.; Bongiorno, A.; Riedo, E. Nat. Mater. 2012, 11, 544-549.

(12) Elias, D. C.; Nair, R. R.; Mohiuddin, T. M. G.; Morozov, S. V.; Blake, P.; Halsall, M. P.; Ferrari, A. C.; Boukhvalov, D. W.; Katsnelson,

M. I.; Geim, A. K.; Novoselov, K. S. Science 2009, 323, 610.

(13) Flores, M. Z. S.; Autreto, P. A. S.; Legoas, S. B.; Galvao, D. S. Nanotechnology 2009, 20, 465704-465706.

(14) Sahin, H.; Ataca, C.; Ciraci, S. Appl. Phys. Lett. 2009, 95, 222510.

(15) Sahin, H.; Ataca, C.; Ciraci, S. Phys. Rev. B 2010, 81, 205417. Sahin, H.; Ciraci, C. Phys. Rev. B 2011, 84, 035452.

(16) Topsakal, M.; Cahangirov, S.; Ciraci, S. Appl. Phys. Lett. 2010, 96, 091912.

(17) Sofo, J. O.; Chaudhari, A. S.; Barber, G. D. Phys. Rev. B 2007, 75, 153401.

(18) Nair, R. R.; Ren, W.; Jalil, R.; Riaz, I.; Kravets, V. G.; Britnell, L.; Blake, P.; Schedin, F.; Mayorov, A. S.; Yuan, S.; Katsnelson, M. I.; Cheng, H.-M.; Strupinski, W.; Bulusheva, L. G.; Okotrub, A. V.; Grigorieva, I. V.; Grigorenko, A. N.; Novoselov, K. S.; Geim, A. K. Small 2010, 6, 2877.

(19) Cheng, S.-H.; Zou, K.; Okino, F.; Gutierrez, H. R.; Gupta, A.; Shen, N; Eklund, P. C.; Sofo, J. O.; Zhu, J. Phys. Rev. B 2010, 81, 205435.

(20) Robinson, J. T.; Burgess, J. S.; Junkermeier, C. E.; Badescu, S. C.; Reinecke, T. L.; Perkins, F. K.; Zalalutdniov, M. K.; Baldwin, J. W.; Culbertson, J. C.; Sheehan, P. E.; Snow, E. S. Nano Lett. 2010, 10, 3001-3005.

(21) Withers, F.; Dubois, M.; Savchenko, A. K. Phys. Rev. B 2010, 82, 073403.

(22) Peelaers, H.; Hernandez-Nieves, A. D.; Leenaerts, O.; Partoens, B.; Peeters, F. M. Appl. Phys. Lett. 2011, 98, 051914.

(23) Sahin, H.; Topsakal, M.; Ciraci, S. Phys. Rev. B 2011, 83, 115432.

(24) Charlier, J.-C.; Gonze, X.; Michenaud, J.-P. Phys. Rev. B 1993, 47, 16152.

(25) Takagi, Y.; Kusakabe, K. Phys. Rev. B 2002, 65, 121103.

(26) Li, B.; Zhou, L.; Wu, D.; Peng, H.; Yan, K.; Zhou, Y.; Liu, Z. F. ACS Nano 2011, 5, 5957-5961.

(27) Wu, J.; Xie, L.; Li, Y.; Wang, H.; Ouyang, Y.; Guo, J.; Dai, H. J. Am. Chem. Soc. 2011, 133, 19668.

(28) Gopalakrishnan, K.; Subrahmanyam, K. S.; Kumar, P.; Govindaraj, A.; Rao, C. N. R. RSC Adv. 2012, 2, 1605.

(29) Wehling, T. O.; Katsnelson, M. I.; Lichtenstein, A. I. Phys. Rev. B 2009, 80, 085428.

(30) Klintenberg, M.; Lebegue, S.; Katsnelson, M. I.; Eriksson, O. Phys. Rev. B 2010, 81, 085433.

(31) Medeiros, P. V. C.; Mascarenhas, A. J. S.; Mota, F. D.; de Castilho, C. M. C. Nanotechnology 2010, 21, 485701.

(32) Zboril, R.; Karlicky, F.; Bourlinos, A. B.; Steriotis, T. A.; Stubos, A. K.; Georgakilas, V.; Safarova, K.; Jancik, D.; Trapalis, C.; Otyepka, M. Small 2010, 6, 2885-2891.

(33) Rudenko, A. N.; Keil, F. J.; Katsnelson, M. I.; Lichtenstein, A. I. Phys. Rev. B 210, 82, 035427.

(34) Dhiman, P.; Yavari, F.; Mi, X.; Gullapalli, H.; Shi, Y.; Ajayan, P. M.; Koratkar, N. Nano Lett. 2011, 11, 3123-3127.

(35) Ijäs, M.; Havu, P.; Harju, A. Phys. Rev. B 2012, 85, 035440.

(36) Yang, M.; Zhou, L.; Wang, J.; Liu, Z.; Liu, Z. J. Phys. Chem. C 2012, 116, 844.

(37) Blochl, P. Phys. Rev. B 1994, 50, 17953-17979.

(38) Ceperley, D.; Alder, B. Phys. Rev. Lett. 1980, 45, 566-569.

(39) Perdew, J. P.; Burke, K.; Ernzerhof, M. Phys. Rev. Lett. 1996, 77, 3865-3868.

(40) Grimme, S. J. Comput. Chem. 2006, 27, 1787-1799.

(41) Kresse, G.; Furthmuller, J. Phys. Rev. B 1996, 54, 11169-11186.

(42) Henkelman, G.; Arnaldsson, A.; Jonsson, H. Comput. Mater. Sci.

2006, 36, 354-360.

(43) Alfe, D. Comput. Phys. Commun. 2009, 180, 2622-2633.

(44) Giannozzi, P.; Baroni, S.; Bonini, N.; Calandra, M.; Car, R.; Cavazzoni, C.; Ceresoli, D.; Chiarotti, G. L.; Cococcioni, M.; Dabo, I.; Corso, A. D.; Gironcoli, S.; Fabris, S.; Fratesi, G.; Gebauer, R.;
Gerstmann, U.; Gougoussis, C.; Kokalj, A.; Lazzeri, M.; Martin-Samos, L.; Marzari, N.; Mauri, F.; Mazzarello, R.; Paolini, S.; Pasquarello, A.; Paulatto, L.; Sbraccia, C.; Scandolo, S.; Sclauzero, S.; Seitsonen, A. P.; Smogunov, A.; Umari, P.; Wentzcovitch, R. M. J. Phys. Condens. Matter 2009, 21, 395502.

(45) Shishkin, M.; Kresse, G. Phys. Rev. B 2006, 74, 035101.

(46) The total energy of magnetic states or nonmagnetic states, whichever is lower, are used in calculating binding, cohesive, and formation energies.

(47) Heyd, J.; Scuseria, G.; Ernzerhof, M. J. Chem. Phys. 2003, 118, $8207-8215$

(48) The experimental value for the binding energy of the $\mathrm{Cl}_{2}$ molecule is $2.52 \mathrm{eV}$ (Haynes, W. M.; Lide, D. R. CRC Handbook of Chemistry and Physics, 91st ed.; CRC: London, UK, 2010, p 57). We also calculated the binding energy of $\mathrm{Cl}_{2}$ using PBE and GGA+vdW to be 3.08 and $3.02 \mathrm{eV}$, respectively. By applying corrections within hybrid functionals (see ref 45) and performing LDA+HSE06, PBE +HSE06, and GGA+HSE06 calculations the binding energies of $\mathrm{Cl}_{2}$ are found to be $3.10,2.65$, and $2.60 \mathrm{eV}$. The latter value calculated with GGA+HSE06 is in excellent agreement with the experimental value. Earlier calculations reported the binding energies of $\mathrm{Cl}_{2}$ to be $3.78 \mathrm{eV}$ [Liu, Z. F.; Chan, S. P. Chem. Phys. Lett. 2000, 318, 15-21], $2.68 \mathrm{eV}$ [Gao, W.; Baker, T. A.; Zhou, L.; Pinnaduwage, D. S.; Kaxiras, E.; Friend, C. M. J. Am. Chem. Soc. 2008, 130, 3560-3565], and 2.54 eV [Broqvist, P.; Moline, L. M.; Gronbeck, H.; Hammer, B. J. Catal. 2004, 227, 217-226]. The LDA method with PAW potential used in the present paper yields overbinding. Since the LDA method is used consistently throughout the paper the overbinding did not affected our conclusions.

(49) Kittel, C. Introduction to Solid State Physics, 7th ed.; Wiley: New York, NY, 1996.

(50) Chan, K. T.; Neaton, J. B.; Cohen, M. L. Phys. Rev. B 2008, 77, 235430.

(51) Sahin, H.; Cahangirov, S.; Topsakal, M.; Bekaroglu, E.; Akturk, E.; Senger, R. T.; Ciraci, S. Phys. Rev. B 2009, 80, 155453.

(52) Yan, J.-A.; Ruan, W. Y.; Chou, M. Y. Phys. Rev. B 2008, 77, 125401.

(53) Topsakal, M.; Akturk, E.; Ciraci, S. Phys. Rev. B 2009, 79, 115442.

(54) Ataca, C.; Sahin, H.; Akturk, E.; Ciraci, S. J. Phys. Chem. C 2011, $115,3934-3941$

(55) Allen, F. G.; Gobeli, G. W. Phys. Rev. 1962, 127, 150.

(56) Ditchfield, R.; Llera-Rodriguez, D.; Seebauer, E. G. Phys. Rev. B 2000, 61 (13), 710. 